\title{
Evaluation Of Epicardial Fat Thickness in Young Patients with Cryptogenic Stroke
}

\author{
Ahmet Gurdal(1); Gozde Baran(2); Kudret Keskin(1); Dilek Necioglu Orken(2); Kadriye \\ Kilickesmez(1)
}

1 Şişli Hamidiye Etfal Research and Training Hospital, Cardiology Clinic; Istanbul, Turkey 2 Şişli Hamidiye Etfal Research and Training Hospital, Neurology Clinic; Istanbul, Turkey

\section{Aim}

Epicardial adipose tissue (EAT) is metabolically active and an important predictor of metabolic and cardiovascular diseases. The aim of this study was to investigate the epicardial adipose tissue measurement with echocardiography in young patients with cryptogenic stroke (CS).

\section{Materials\&Method}

We studied 77 volunteers: 40 patients with CS (mean age $43 \pm 8$ years,16 females) and other 37 healthy subjects (mean age $38 \pm 7$ years, 20 females). All basic biochemical parameters were analyzed and echocardiographic epicardial fat thickness (EFT) were measured in all subjects. All imaging and examinations were done in terms of etiology in the patients. The categorical and numerical variables were compared using the chi-square test and independent t-test, respectively. Correlations were analyzed using the Spearman and Pearson correlation tests. ROC curve analysis was performed to determine cutoff high risk value of EFT.

\section{Results}

In comparison with the control group, the patients with CS had significantly higher EFT $(5.51 \pm 0.82,3.96 \pm 0.51, \quad p<0.01)$. Furthermore, there were positive correlations between EFT and C-reaktive protein $(r=0.284 ; p<0.05)$. A cutoff high risk EFT value of $4.6 \mathrm{~mm}$ showed a sensitivity and specificity of $87.5 \%$ and $81.1 \%$, respectively.

\section{Conclusion}

We found that echocardiographic EFT was significantly higher in young patients with CS. Increased EFT might be a novel risk factor in these patients.

Table 1: Demographic and biochemical parameters of the study population

\begin{tabular}{|c|c|c|c|}
\hline & $\begin{array}{l}\text { Controls } \\
(n=37)\end{array}$ & $\begin{array}{l}\text { ESUS } \\
(n=40)\end{array}$ & $P$ value \\
\hline Age (year) & $38 \pm 7$ & $43 \pm 8$ & 0.072 \\
\hline Sex (male/female) & $17 / 20$ & $24 / 16$ & 0.217 \\
\hline $\mathrm{BMI}\left(\mathrm{kg} / \mathrm{m}^{2}\right)$ & $25.8 \pm 3.1$ & $26.2 \pm 3.4$ & 0.443 \\
\hline Smoking & $13 / 24$ & $28 / 12$ & 0.002 \\
\hline $\mathrm{SBP}(\mathrm{mmHg})$ & $113 \pm 9$ & $121 \pm 11$ & 0.281 \\
\hline $\mathrm{DBP}(\mathrm{mmHg})$ & $72 \pm 5$ & $75 \pm 7$ & 0.140 \\
\hline Cholesterol (mg/dL) & $192 \pm 33$ & $193 \pm 48$ & 0.168 \\
\hline Triglycerides $(\mathrm{mg} / \mathrm{dL})$ & $123 \pm 53$ & $156 \pm 80$ & 0.323 \\
\hline $\mathrm{HDL}(\mathrm{mg} / \mathrm{dL})$ & $49 \pm 10$ & $43 \pm 13$ & 0.705 \\
\hline $\mathrm{LDL}(\mathrm{mg} / \mathrm{dL})$ & $115 \pm 31$ & $117 \pm 44$ & 0.480 \\
\hline Creatinine $(\mathrm{mg} / \mathrm{dL})$ & $0.72 \pm 0.12$ & $0.79 \pm 0.18$ & 0.052 \\
\hline Hemoglobin (g/dL) & $13.5 \pm 1.7$ & $13.8 \pm 1.9$ & 0.561 \\
\hline Glucose (mg/dL) & $88 \pm 8$ & $93 \pm 13$ & 0.064 \\
\hline Fibrinogen(mg/dL) & $270 \pm 37$ & $315 \pm 61$ & 0.054 \\
\hline C-reactive protein $(\mathrm{mg} / \mathrm{dL})$ & $3.2 \pm 1.1$ & $4.2 \pm 2.4$ & 0.002 \\
\hline
\end{tabular}

*Values are presented as mean \pm SD. ESUS:Embolic Stroke of Undetermined Source_BMI:Body Mass Index; SBP:Systolic Blood Pressure; DBP:Diastolic Blood Pressure; HDL:High Density Lipoprotein; LDL:Low Density Lipoprotein

Table 2: The echocardiographic characteristics of the groups

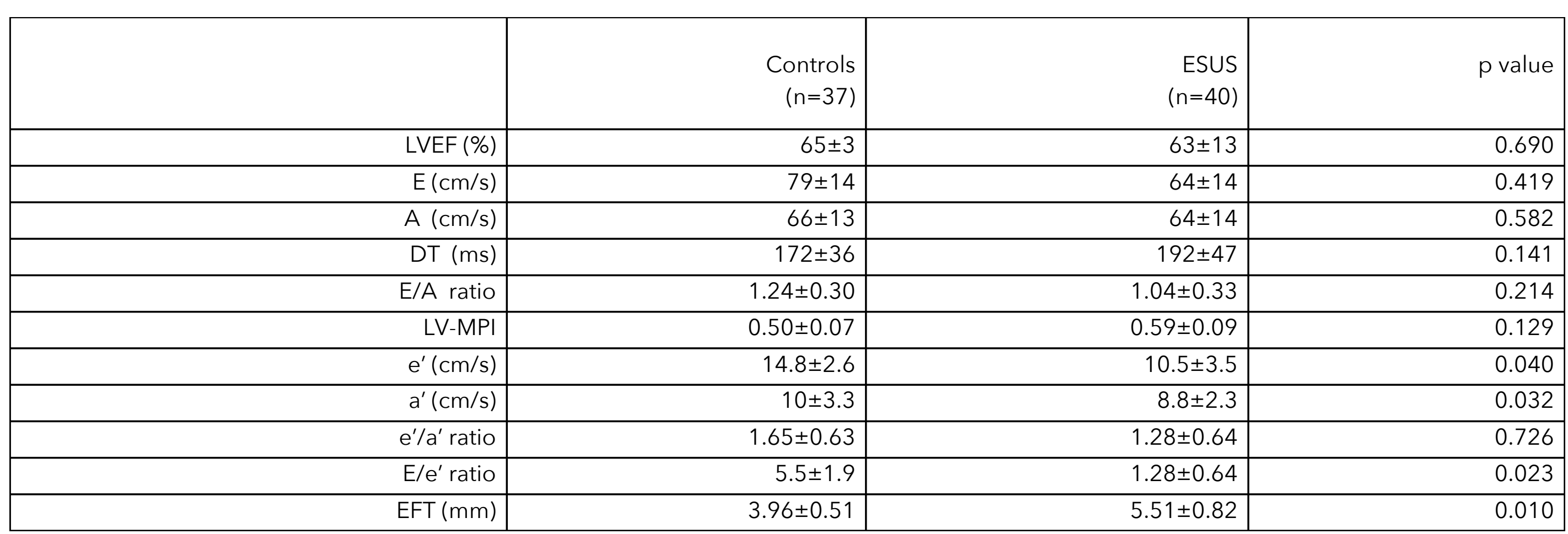

Values are presented as mean $\pm S D$, ESUS:Embolic Stroke of Undetermined Source; LVEF: Left ventricular ejection fraction; E:Peak early filling velocity; A:Late diastolic filling velocity; DT:E wave deceleration time, LV-MPI:Left ventricular myocardial performance index; e': early diastolic mitral annular velocity; a': late diastolic mitral annular 